\title{
Link protein hyaluronan-binding motif abrogates CD44-hyaluronan-mediated leukemia-liver cell adhesion
}

\author{
JING CHEN, NA LI and GONGCHU LI \\ College of Life Sciences, Zhejiang Sci-Tech University, Zhejiang 310018, P.R. China
}

Received September 6, 2012; Accepted January 2, 2013

DOI: $10.3892 / \mathrm{mco} .2013 .73$

\begin{abstract}
The liver is a frequent site for the metastasis of cancer cells originating from other sites. Leukemic liver metastasis is associated with poor prognosis. The ligation of CD44 with hyaluronan (HA) has been shown to contribute to the drug resistance of leukemic cells. In this study, a link protein HA-binding motif was genetically fused with enhanced green fluorescence protein (EGFP) to generate an EGFP-L fusion protein. Furthermore, a coculture system was established to investigate the interaction of leukemic cells with liver cells. CD44-positive Kasumi-1, but not CD44-negative HL-60 cells, were observed to adhere to the liver cell line L02. This cell-cell adhesion was significantly blocked by HA, indicating that Kasumi-L02 cell adhesion was mediated by the CD44-HA interaction. Compared to EGFP, EGFP-L fusion protein bound to L02 and BEL7404 liver cells. EGFP-L partially abrogated the Kasumi-L02 adhesion, suggesting that the link protein-binding motif is able to inhibit CD44-HA-mediated leukemia-liver adhesion. These results may help provide insight into novel therapeutic methods for leukemic patients diagnosed with liver metastasis.
\end{abstract}

\section{Introduction}

The liver is a frequent site for the metastasis of cancer cells originating from other sites. A significant number of leukemic or lymphoma patients have liver metastasis (1). Notably, liver enlargement with malignant infiltration in leukemic and lymphoma patients is associated with poor prognosis (2). Inhibition of the interaction between liver cells and infiltrated malignant cells may provide a novel therapeutic method for patients diagnosed with leukemic liver metastasis.

The CD44 transmembrane glycoprotein is ubiquitously expressed in cells and tissues and is involved in inflammation (3) and tumorigenesis (4). Due to alternative splicing of various

Correspondence to: Dr Gongchu Li, College of Life Sciences, Zhejiang Sci-Tech University, 2nd Street, Xia Sha, Hangzhou, Zhejiang 310018, P.R. China

E-mail: lgc@zstu.edu.cn

Key words: leukemia, liver metastasis, CD44, hyaluronan, cell-cell adhesion exons, CD44 is usually expressed in a variety of isoforms (CD44v) (5). The CD44 isoforms contain a hyaluronan (HA)binding domain in the extracellular domain (6). HA is a major component of the extracellular matrix (ECM), and interacts with a variety of ECM and cell membrane proteins (6-10), among which link protein is a component of ECM interacting with HA through its HA-binding motif (16). As an important cell adhesion molecule, CD44 mediates cell-cell and cell-ECM interaction, inducing intracellular signaling events $(11,12)$. Notably, the ligation of CD44 has been shown to induce the activation or expression of intracellular signaling elements, such as MDR1, phosphoinositide 3 kinase (PI3K) (13), Lyn (14), $\mathrm{Ca}^{2+}$ mobilization (15) and ERK (16), which are involved in apoptosis inhibition and chemoresistance in tumors.

In this study, an in vitro coculture system of leukemic and liver cells was established, determined to be mediated by the interaction of CD44 and HA. A fusion protein containing enhanced green fluorescent protein (EGFP) and a link protein HA-binding motif (EGFP-L) were used to detect cell membrane HA on liver cells and block the adhesion of CD44-positive leukemia with liver cells.

\section{Materials and methods}

Cells. The Kasumi-1 human acute myeloid leukemia cell line was kindly provided by Professor S.J. Chen (Shanghai Jiaotong University, Shanghai, China) and maintained in RPMI-1640 medium (Hyclone Laboratories, Inc., Logan, UT, USA) supplemented with $20 \%$ fetal bovine serum (FBS) (Life Technologies, Inc., Grand Island, NY, USA) and 1\% L-glutamine (Life Technologies, Inc.). The HL-60 human acute myeloid leukemia cell line was purchased from the American Type Culture Collection (ATCC; Manassas, VA, USA) and maintained in RPMI-1640 medium (Hyclone Laboratories, Inc.) supplemented with $10 \%$ FBS (Life Technologies, Inc.) and $1 \%$ L-glutamine (Life Technologies, Inc.). The L02 human fetal liver cell line and the BEL7404 human hepatoma cell line were purchased from the Shanghai Cell Collection (Shanghai, China) and cultured in Dulbecco's modified Eagle's medium (DMEM) (Invitrogen, Carlsbad, CA, USA), supplemented with $10 \%$ FBS (Life Technologies, Inc.) and 1\% L-glutamine (Life Technologies, Inc.).

Plasmid construction and recombinant protein preparation. A sequence encoding EGFP was amplified from plasmid 
A

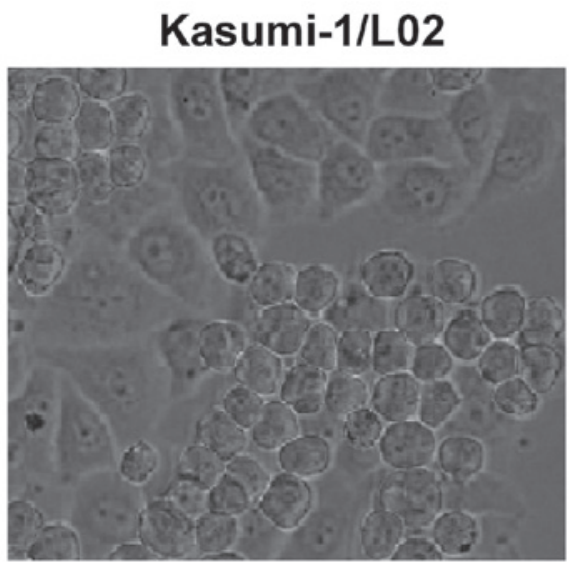

HL-60/L02

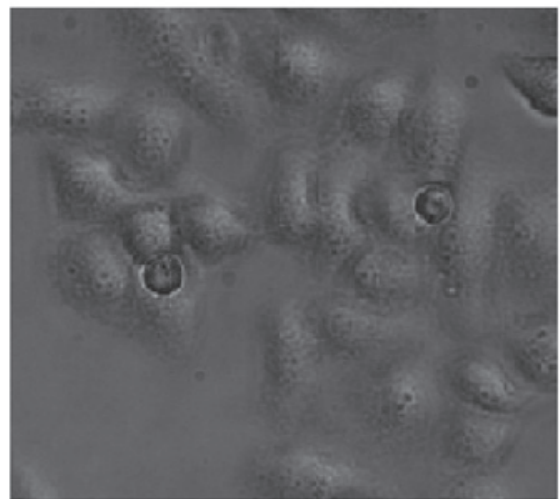

B

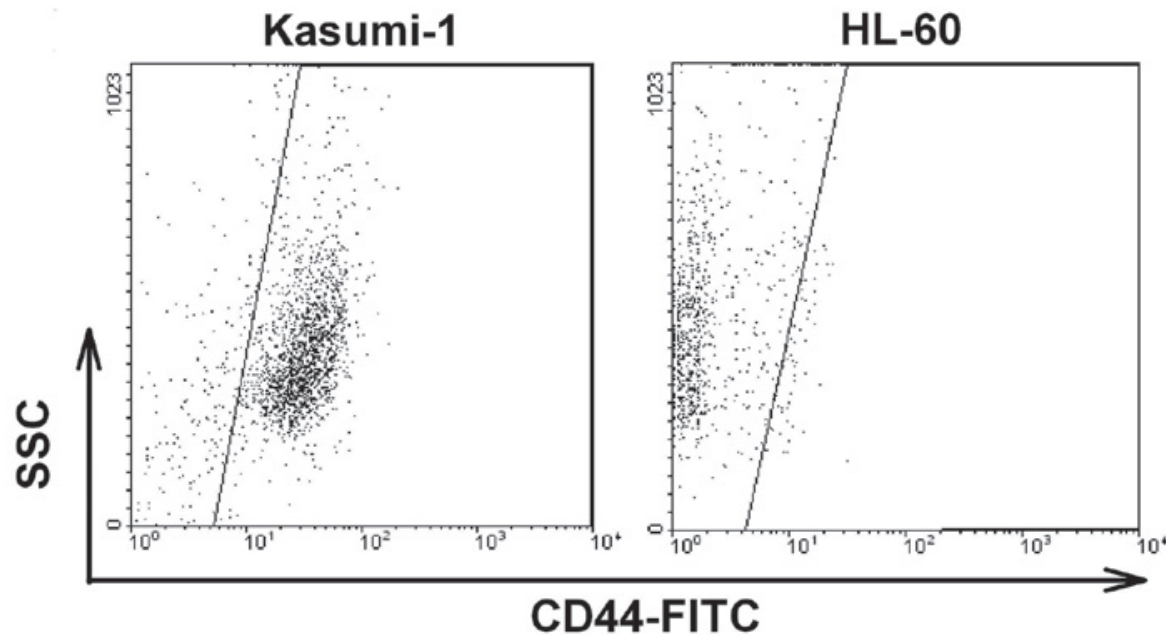

Figure 1. Adhesion of CD44-positive Kasumi-1 cells, but not CD44-negative HL-60 cells with liver cells. (A) Adherence of Kasumi-1 or HL-60 cells with L02 liver cells. Kasumi-1 or HL-60 cells were cocultured with L02 cells and then washed with PBS. Adherent cells were observed using a microscope (magnification, x200). Data are representative of three independent experiments. (B) Flow cytometric analysis of the CD44 expression of Kasumi-1 and HL-60 cells. SSC, subsurface cisternae; CD44-FITC, CD44-fluorescein isothiocyanate.

pEGFP-C1 using PCR. The sense primer contained an NdeI site (5'-TATCATATGGTGAGCAAGGGCGAG-3'), while the antisense primer contained an XhoI site (5'-TATCT CGAGTT AGCATCTGAGTCC-3'). The EGFP-L fusion gene containing a sequence encoding EGFP and a link protein HA-binding motif with the amino acid sequence ${ }^{316}$ RYPISRPRKR ${ }^{325}$ (17) was generated by PCR from pEGFP-C1. The sense primer contained an NdeI site (5'-TAT CATATGGTGAGCA AGG GCGAG-3'), while the antisense primer contained a sequence encoding the link protein HA-binding motif and an XhoI site (5'-TATCTCGAG TTAGCGCTTTCTGGGTCTGGAGATGGGGTAGCGAGA TCTGAGTCCGGACT-3'). PCR products were inserted into the corresponding site of plasmid pET-28 $\alpha$ to generate plasmids pET-28 $\alpha$-EGFP and pET-28 $\alpha$-EGFP-L.

The plasmid pET-28 $\alpha$-EGFP or pET-28 $\alpha$-EGFP-L was transformed into Escherichia coli strain M15 and the expression of recombinant proteins was induced by $0.08 \mathrm{mM}$ of isopropyl- $\beta$-D-thiogalactopyranoside (IPTG) at room temperature for $6 \mathrm{~h}$. Cells were harvested by centrifugation at 7,104 x g for $15 \mathrm{~min}$ followed by resuspension in PBS and disruption by sonication. Bacterial debris was pelleted by centrifugation at $15,984 \mathrm{x}$ g for $15 \mathrm{~min}$. Supernatants were mixed with Ni-NTA slurry (Merck Biosciences, Darmstadt, Germany). The lysate-Ni-NTA mixture was loaded into a column and washed twice with a washing buffer containing $300 \mathrm{mM} \mathrm{NaCl}, 50 \mathrm{mM}$ sodium phosphate buffer and $20 \mathrm{mM}$ imidazole ( $\mathrm{pH}$ 8.0). The column was eluted with an elution buffer containing $300 \mathrm{mM} \mathrm{NaCl}, 50 \mathrm{mM}$ sodium phosphate buffer and $250 \mathrm{mM}$ imidazole ( $\mathrm{pH}$ 8.0). The eluted protein was dialyzed against PBS at $4{ }^{\circ} \mathrm{C}$ overnight to remove imidazole. Proteins $(\sim 30 \mathrm{kDa})$ were then analyzed by SDS-PAGE followed by Coomassie Brilliant Blue staining. The protein concentration was determined using the BCA Protein Assay kit (Thermo Fisher Scientific, Inc., Rockford, IL, USA).

Flow cytometry. For the detection of CD44 expression, Kasumi-1 or HL-60 cells were incubated with fluorescein isothiocyanate (FITC)-conjugated anti-CD44 antibody (BD Biosciences, San Jose, CA, USA) at $4^{\circ} \mathrm{C}$ for $20 \mathrm{~min}$. FITC-conjugated mouse IgG staining served as the control. Following staining, the cells were analyzed on a BD FACSAria flow cytometer (BD Biosciences).

EGFP-L staining analysis. L02 or BEL7404 cells were cultured in $24-w e l l$ plates. When cells reached $60-70 \%$ conflu- 


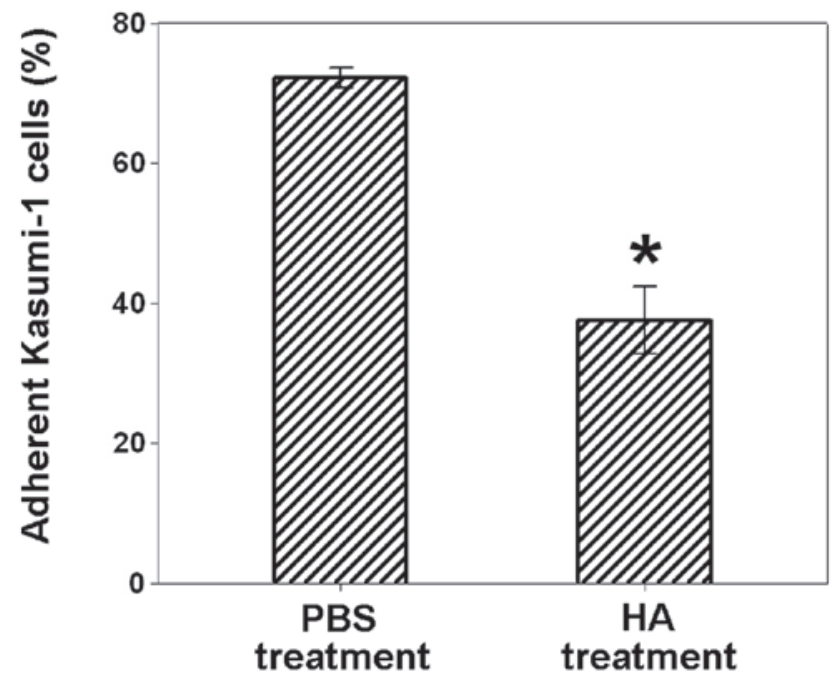

Figure 2. Adhesion of Kasumi-1 cells with liver cells was blocked by hyaluronan (HA). Kasumi-1 cells were pretreated with HA followed by coculture with L02 cells. Phosphate-buffered saline (PBS) pretreatment served as the control. Non-adherent Kasumi-1 cells were collected and counted. The percentage of adherent Kasumi-1 cells was calculated. Values are presented as the mean \pm standard error of the mean (SEM) from three separate experiments. ${ }^{\prime} \mathrm{P}<0.05$ compared to the control.

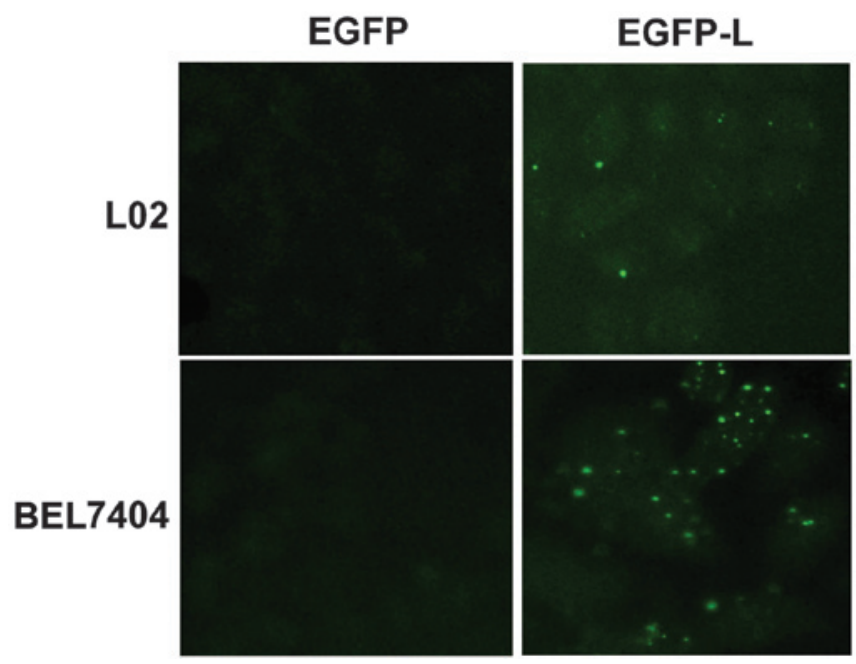

Figure 3. Detection of the liver cell hyaluronan (HA), using enhanced green fluorescent protein and a link protein HA-binding motif (EGFP-L) staining. L02 or BEL7404 liver cells were cultured followed by EGFP-L staining. EGFP served as the control. Green fluorescence was observed using a fluorescence microscope (magnification, x200).

ence, the culture medium was aspirated and $150 \mu \mathrm{g} / \mathrm{ml}$ of EGFP-L was added. Following $20 \mathrm{~min}$ of incubation at $4^{\circ} \mathrm{C}$, EGFP-L was removed. Cells were then washed with PBS three times. EGFP staining served as the control. A fluorescent microscope (Olympus Corporation, Tokyo, Japan) was used to observe green fluorescence.

Cell-cell adhesion assay. L02 cells were cultured in 24-well plates. When cells reached $60-70 \%$ confluence, the culture medium was aspirated, and Kasumi-1 or HL-60 cells were added. After 30-45 min, any Kasumi-1 or HL-60 cells that

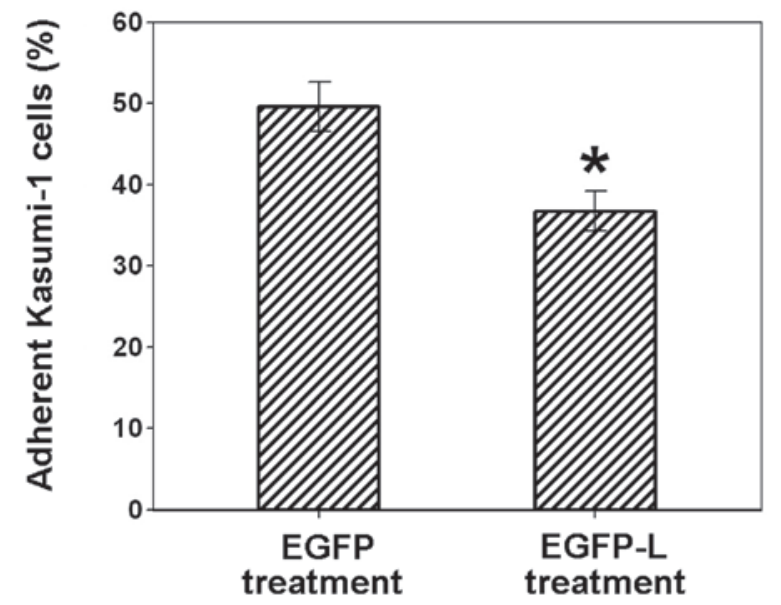

Figure 4. Adhesion of Kasumi-1 cells with L02 cells was blocked by enhanced green fluorescent protein and a link protein hyaluronan (HA)-binding motif (EGFP-L). L02 cells were cultured and pretreated with EGFP-L followed by coculture with Kasumi-1 cells. EGFP pretreatment was used as the control. Non-adherent Kasumi-1 cells were collected and counted. The percentage of adherent Kasumi-1 cells was calculated. Values are presented as the mean \pm standard error of the mean (SEM) from four separate experiments. $\mathrm{P}<0.05$.

were non-adherent to L02 cells were removed. A microscope (Olympus Corporation) was used to observe cell-cell adhesion.

For the HA blocking experiments, Kasumi-1 cells were incubated with $1 \mathrm{mg} / \mathrm{ml}$ of $\mathrm{HA}$ at $4^{\circ} \mathrm{C}$ for $10 \mathrm{~min}$. Cells were then added to the same volume of culture medium followed by coculture with L02 precultured in 24-well plates. After 30 min, Kasumi-1 cells that were non-adherent to L02 cells were collected and counted. PBS treatment served as the control.

For the EGFP-L blocking experiments, L02 cells cultured in 24-well plates were incubated with $150 \mu \mathrm{g} / \mathrm{ml}$ of EGFP-L or EGFP at $4^{\circ} \mathrm{C}$ for $30 \mathrm{~min}$. Kasumi-1 cells were then added. After 45 min, Kasumi-1 cells that were non-adherent to L02 cells were collected and counted.

Statistical analysis. Differences among the treatment groups were assessed by ANOVA. $\mathrm{P}<0.05$ was considered to indicate a statistically significant difference.

\section{Results}

Leukemic cells adhere to liver cells through CD44. The Kasumi-1 leukemia cell line adhered to L02 liver cells. However, this cell-cell adhesion did not occur in HL-60 cells (Fig. 1A). By analyzing the expression of cell membrane proteins, we determined that the majority of the Kasumi-1 cells expressed CD44, and most of the HL-60 cells had a CD44-negative phenotype (Fig. 1B). Therefore, we hypothesized that leukemic cells may adhere to L02 cells through CD44. To examine this hypothesis, HA, a natural ligand for CD44 was used to pretreat Kasumi-1 cells followed by the coculture of Kasumi-1 with L02 cells. As shown in Fig. 2, the treatment of Kasumi-1 cells with HA significantly blocked the adhesion of Kasumi-1 with L02 cells (37.6 $\pm 4.8 \%$ of adherent Kasumi-1 cells), compared to the PBS control 
$(72.3 \pm 1.4 \%$ of adherent Kasumi-1 cells). These results demonstrated that CD44 is involved in the adhesion of leukemic cells with liver cells.

Liver cells express HA on the cell membrane. HA is a linear glycosaminoglycan with the molecular mass of a few million Daltons and can be secreted by cells to the ECM or associated with the plasma membrane to form a pericellular coat (18). Certain cell-associated and ECM proteins, such as CD44, link protein and collagen VI (6-10) have been found to mediate the biological functions of HA. To determine the liver cell membrane component involved in the adhesion with CD44-positive leukemia cells, EGFP-L, a fusion protein containing EGFP and a link protein HA-binding motif were generated to detect the cell membrane HA on liver cells. Recombinant EGFP was used as the control. The majority of L02 and BEL7404 cells were labeled with EGFP-L as compared to the EGFP control, indicating that liver cells L02 and BEL7404 expressed HA on the cell membrane (Fig. 3). Notably, EGFP-L formed bright dots on the cell membrane of L02 and BEL7404 cells, suggesting that HA may associate with certain special membrane structures in liver cells. Taken together, these results demonstrated that the CD44-HA interaction was involved in the adhesion of leukemic cells with liver cells.

EGFP-L abrogates the adhesion of leukemic and liver cells. To determine the effect of EGFP-L on the adhesion of Kasumi-1 with L02 cells, L02 cells were pretreated with EGFP-L and then cocultured with Kasumi-1 cells. Recombinant EGFP was used as the control. As shown in Fig. 4, treatment of L02 cells with EGFP-L significantly blocked the adhesion of L02 cells with Kasumi-1 cells (36.8 $\pm 2.5 \%$ of adherent Kasumi-1 cells), compared to EGFP treatment $(49.6 \pm 3.0 \%$ of adherent Kasumi-1 cells). These results indicate that the link protein HA-binding motif is effective in inhibiting the adhesion between CD44-positive leukemia cells with HA-positive liver cells.

\section{Discussion}

Ligation of CD44 with HA is associated with the drug resistance of cancer cells. Furthermore, CD44-HA interaction activates ErbB2 and PI3K to stimulate multidrug transporter MDR1, resulting in the drug resistance of breast and ovarian cancer cells $(13,19)$. CD44 and MDR1 have been found to be coexpressed in small cell lung cancer chemoresistant cells (20). Drug resistance induced by the CD44-HA interaction has also been observed in head and neck cancer $(15,16)$. Notably, the ligation of CD44 with monoclonal antibodies has been demonstrated to decrease drug-induced apoptosis in acute myeloid leukemia cells (21). The results of this study have shown that the link protein HA-binding motif bound with HA in liver cell membranes and abrogated the adhesion of CD44-positive leukemia cells with liver cells. These findings may provide insight into developing novel methods to inhibit liver metastasis in leukemia.

\section{Acknowledgements}

This study was supported by the National Natural Science Foundation of China (30801379).

\section{References}

1. Scheimberg IB, Pollock DJ, Collins PW, et al: Pathology of the liver in leukaemia and lymphoma. A study of 110 autopsies. Histopathology 26: 311-321, 1995.

2. Frei E III, Fritz RD, Price E, et al: Renal and hepatic enlargement in acute leukemia. Cancer 16: 1089-1092, 1963.

3. Khan AI, Kerfoot SM, Heit B, et al: Role of CD44 and hyaluronan in neutrophil recruitment. J Immunol 173: 7594-7601, 2004.

4. Naor D, Sionov RV and Ish-Shalom D: CD44: structure, function, and association with the malignant process. Adv Cancer Res 71: 241-319, 1997.

5. Screaton GR, Bell MV, Jackson DG, et al: Genomic structure of DNA encoding the lymphocyte homing receptor CD44 reveals at least 12 alternative spliced exons. Proc Natl Acad Sci USA 89: 12160-12164, 1992.

6. Underhill C: CD44: the hyaluronan receptor. J Cell Sci 103: 293-298, 1992.

7. Deak F, Kiss I, Sparks KJ, et al: Complete amino acid sequence of chicken cartilage link protein deduced from cDNA clones. Proc Natl Acad Sci USA 83: 3766-3770, 1986.

8. Turley EA, Moore D and Hayden LJ: Characterization of hyaluronate binding proteins isolated from $3 \mathrm{~T} 3$ and murine sarcoma virus transformed 3T3 cells. Biochem 26: 2997-3005, 1987.

9. Doege KJ, Sasaki M, Kimura T and Yamada Y: Complete coding sequence and deduced primary structure of the human cartilage large aggregating proteoglycan, aggrecan. J Biol Chem 266: 894-902, 1991

10. McDevitt CA, Marcelino J and Tucker L: Interaction of intact type VI collagen with hyaluronan. FEBS Lett 294: 167-170, 1991.

11. Ponta H, Sherman L and Herrlich PA: CD44: from adhesion molecules to signaling regulators. Nat Rev Mol Cell Biol 4: 33-45, 2003.

12. Thorne RF, Legg JW and Isacke CM: The role of the CD44 transmembrane and cytoplasmic domains in co-ordinating adhesive and signaling events. J Cell Sci 117: 373-380, 2004.

13. Misra S, Ghatak S and Toole BP: Regulation of MDR1 expression and drug resistance by a positive feedback loop involving hyaluronan, phosphoinositide 3-kinase, and ErbB2. J Biol Chem 280: 20310-20315, 2005.

14. Bates RC, Edwards NS, Burns GF, et al: A CD44 survival pathway triggers chemoresistance via Lyn kinase and phosphoinositide 3-kinase/Akt in colon carcinoma cells. Cancer Res 61: 5275-5283, 2001.

15. Wang SJ and Bourguignon LY: Hyaluronan-CD44 promotes phospholipase $\mathrm{C}$-mediated $\mathrm{Ca}^{2+}$ signaling and cisplatin resistance in head and neck cancer. Arch Otolaryngol Head Neck Surg 132: 19-24, 2006.

16. Wang SJ and Bourguignon LY: Hyaluronan and the interaction between CD44 and epidermal growth factor receptor in oncogenic signaling and chemotherapy resistance in head and neck cancer. Arch Otolaryngol Head Neck Surg 132: 771-778, 2006.

17. Yang B, Yang BL, Savani RC and Turley EA: Identification of a common hyaluronan binding motif in the hyaluronan binding proteins RHAMM, CD44, and link protein. EMBO J 13: 286-296, 1994.

18. Cohen M, Klein E, Geiger B and Addadi L: Organization and adhesive properties of hyaluronan pericellular coat of chondrocytes and epithelial cells. Biophys J 85: 1996-2005, 2003.

19. Bourguignon LY, Peyrollier K, Xia W and Gilad E: HyaluronanCD44 interaction activates stem cell marker Nanog, Stat-3-mediated MDR1 gene expression, and ankyrin-regulated multidrug efflux in breast and ovarian tumor cells. J Biol Chem 283: 17635-17651, 2008

20. Gutova M, Najbauer J, Gevorgyan A, et al: Identification of uPAR-positive chemoresistant cells in small cell lung cancer. PLoS One 2: e243, 2007.

21. Allouche M, Charrad RS, Bettaieb A, et al: Ligation of the CD44 adhesion molecule inhibits drug-induced apoptosis in human myeloid leukemia cells. Blood 23: 1187-1190, 2000. 